\title{
EVALUATION OF CHEST X -RAYS IN CHILDREN WITH ACUTE WHEEZING
}

\author{
Slobodanka PETROVIĆ ${ }^{1,2}$, Gordana VILOTIJEVIĆ DAUTOVIĆ ${ }^{1,2}$, Branislavka BJELICA RODIĆ \\ Nenad BARIŠIĆ ${ }^{1,2}$, Sanela DOMUZ ${ }^{3}$
}

\author{
${ }^{1}$ University of Novi Sad, Faculty \\ of Medicine, Novi Sad, Serbia \\ ${ }^{2}$ Institute for Child and Youth Health \\ of Vojvodina, Novi Sad, Serbia \\ ${ }^{3}$ School of Applied Medical Sciences \\ Prijedor, Bosnia and Herzegovina
}

\section{Corresponding author: \\ Nenad Barišić \\ Ive Andrica 1 \\ 21000 Novi Sad \\ Serbia \\ bakiboy@orion.rs \\ Tel.: + 381642115233}

Fax.: + 38121420452

Received: November 23, 2013

Accepted: May 4, 2013

Copyright (C) 2013 by University Clinical Center Tuzla. E-mail for permission to publish: paediatricstoday@ukctuzla.ba

\begin{abstract}
This paper was designed as a review of different CXR presentations in our asthmatic patients, or children with wheezing caused by other pathological conditions. Wheezing and asthma are common in childhood. Wheezing is just one of the symptoms of asthma, but also may be a sign associated with a number of various and sometimes very serious diseases. All pediatricians should bear in mind that "not all that wheezes is asthma". It is estimated that approximately $30 \%$ of children have episodes of wheezing or asthma at some stage. Physicians frequently order chest X-rays (CXR) for suspected asthmatic children or severe asthmatic children in acute attacks, in children with wheezing who are less than 5 years of age, for those with a temperature greater than $38.3^{\circ} \mathrm{C}$, those with symptoms for longer than few days, or tachypnea and dyspnea. Retrospective reviews of CXR in children with asthma and wheezing disorders suggest than in most circumstances this investigation does not contribute to diagnosis and management. However, CXR may be of use in identifying complications of asthma or recognizing other wheezing disorders. Unrecognized causes of wheezing may cause serious complications and even fatal outcome. Conclusion - An efficient approach in patients with wheezing would be to forego CXR when a clinical diagnosis is certain, and to perform them only if a complication is suspected or if the etiology of the respiratory illness is uncertain.
\end{abstract}

Key words: Chest X ray • Childhood wheezing • Diagnose - Asthma.

\section{Introduction}

Not all the children who wheeze have asthma. Only $30 \%$ of infants who wheeze go on to develop asthma (1). Several diseases, both common and not so common, can cause wheezing. Chest $\mathrm{x}$-rays (CXRs) are frequently performed for evaluation of respiratory distress. Routine radiography does not need to be part of the initial routine workup of asthma. An efficient and cost-effective approach would be to forego CXR when the clinical diagnosis is certain and to perform one only if a complication is suspected or if the etiology of the respiratory illness is uncertain.

Although it is "traditional" to perform CXR in all children during their first episode of wheezing, a recent study looking at physicians' practices suggests that films are not routinely obtained in all cases (2). The majority of patients presenting with wheezing can be diagnosed clinically by medical history 
and physical examination. A CXR is the most commonly used criteria for defining pneumonia, but also has a place in the diagnostic process of persistent wheezing in childhood (3). In children with wheezing disorders, including severe asthma attacks, CXR can reveal different findings: generalized or unilateral hyperinflation, localized disturbance of ventilation, patchy or irregular aeration (alternating areas of atelectasis and air trapping), peribronchial thickening and bronchial wall thickening. Also, radiographs may provide evidence of a foreign body, associated vascular anomalies, cardiac enlargement, pulmonary hypertension, infiltrates, cysts, or masses. Signs and symptoms that are most likely to be associated with pathological findings on CXR include prolonged fever and localized wheezing or riles by auscultation (4).

Many physicians order CXRs for pediatric patients who present with wheezing despite the paucity of research to support this testing, which exposes patients to radiation, causes delays in therapy and augments the costs. On the other hand, although in an infant or a young child with persistent or re- current wheezing the probability of asthma is high, one must remain very careful in order not to miss other possible causes. Selected cases show different pathological conditions in children that are usually associated with wheezing, and where, in our opinion, radiographic assessment is justified.

The aim of this paper is to present different radiological findings in children (1-18 years) with persistent or recurrent wheezing, to stress the role and significance of CXRs in the diagnostic algorithm of wheezing disorders in childhood, and to point out when it is reasonable and justifiable to carry out radiological evaluation in wheezing children.

\section{Case reviews \\ Chest radiograph in evaluation of asthma attacks}

Most wheezers will not have positive radiographs, but careful clinical evaluation should reveal which patients will have abnormal radiographs and will therefore benefit from the procedure. Therefore, in those patients, it seems reasonable or sensible to obtain CXRs
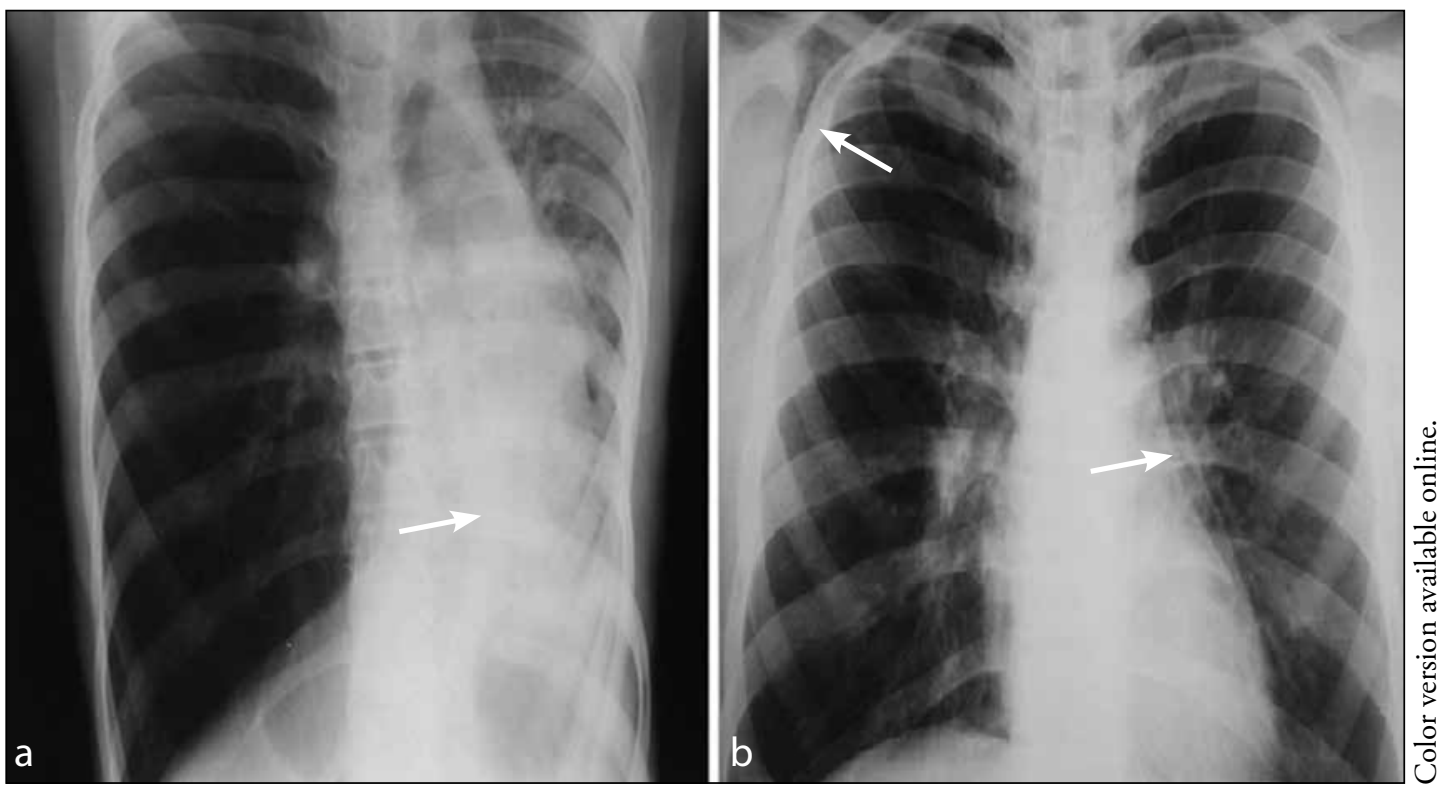

Fig. 1 Asthma complications ( $a$-Status asthmaticus with atelectasis of the left lower lobe and mediastinal shift to the left; $b$-Status asthmaticus with pneumomediastinum and emphysema subcutaneous). 
only if there is a suspicion of complications of asthma, such are pneumothorax, emphysema, concomitant pneumonia, etc. Fig. 1 shows CXRs of two patients with clear diagnosis of allergic asthma, who were hospitalized during severe acute asthma attacks. In these patients, CXRs were necessary because of the severity of the respiratory distress.

The CXR of the first child (Fig. 1a) showed atelectasis of the left lower lobe. Bronchoscopy was performed, and after removing a mucous plug, the respiratory status and radiological findings improved. The second patient (Fig. 1b) is adolescent, with a history of pure controlled asthma from early childhood. He also had a severe asthma attack, complicated by subcutaneous emphysema and mediastinal air leakage (Fig. 1b).

\section{Chest radiograph in evaluation of wheezing in patients with suspected foreign body aspiration (FB)}

Over a 10 year period we had 33 patients with unrecognized foreign body aspiration (FBA). In these cases, FBA presented with persistent wheezing unresponsive to bronchodilatators in $54.5 \%$ of the patients. Wheezing and pulmonary infiltrates were present in $12.1 \%$ of the patients, and other pulmonary complica-
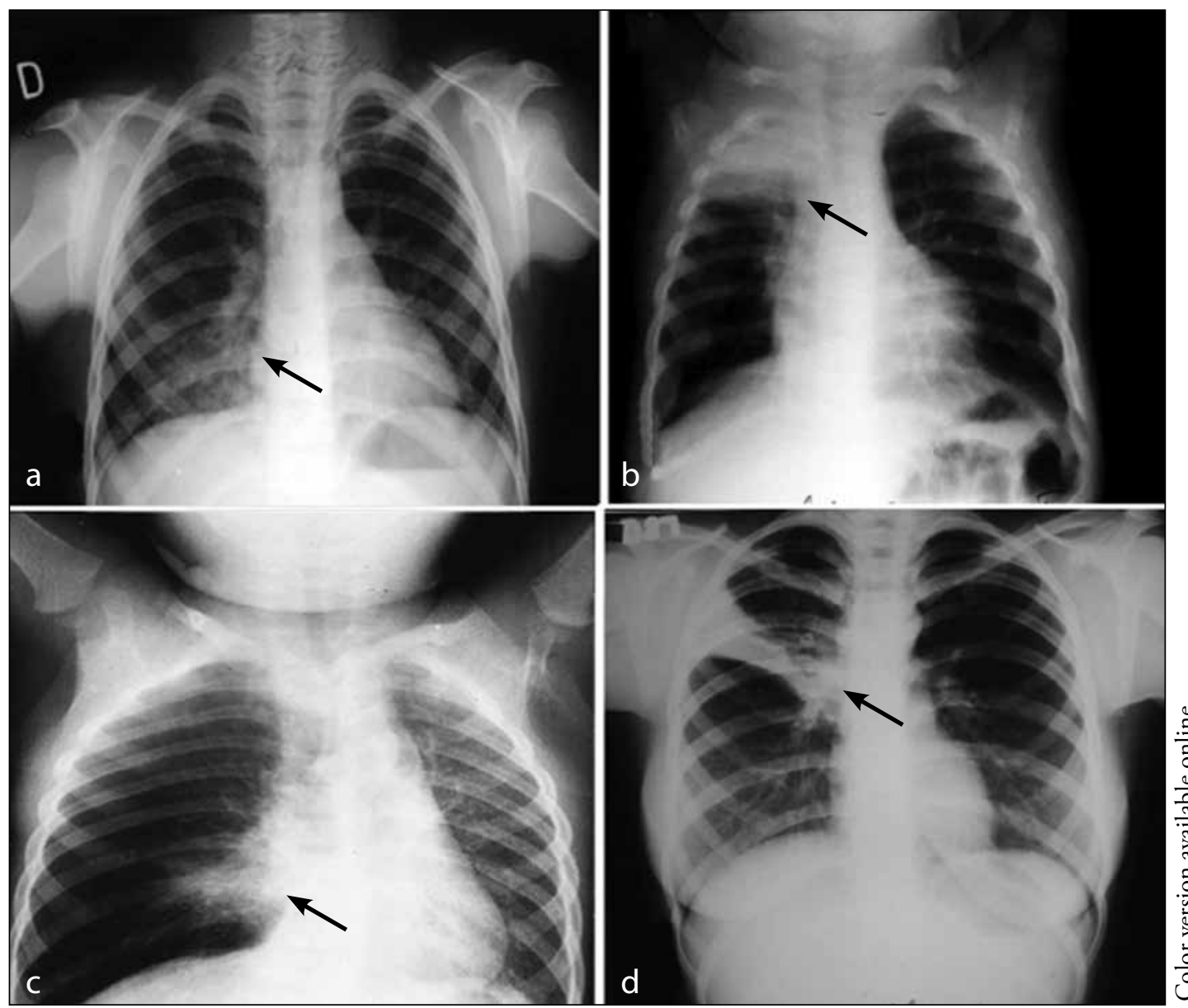

Fig. 2 Foreign body aspirations-different radiological presentations ( $a$ - pneumonia lower lobe, right side; $b$ atelectasis of the superior lobe, right side; $c$ - localized disturbances of ventilation on right side (hyperinflation of the most part of right lobe with infiltrates and atelectasis of middle lobe); $d$-segmental atelectasis on the right). 
tions (persistent pulmonary infections, bronchiectasiae, etc.) in the remaining patients. Delay between FBA and admission to hospital was between 10 days ( $48.5 \%$ of patients) and more than 8 weeks (30.3\% of patients). All children with unrecognized FBA had pathological CXRs, with different presentations (Fig. 2). However, a normal chest $\mathrm{x}$-ray does not rule-out the presence of a foreign body.

\section{Chest radiograph in evaluation of wheezing in patients with bronchiolitis}

Significantly abnormal chest radiographs (focal atelectasis, pulmonary infiltrates, air

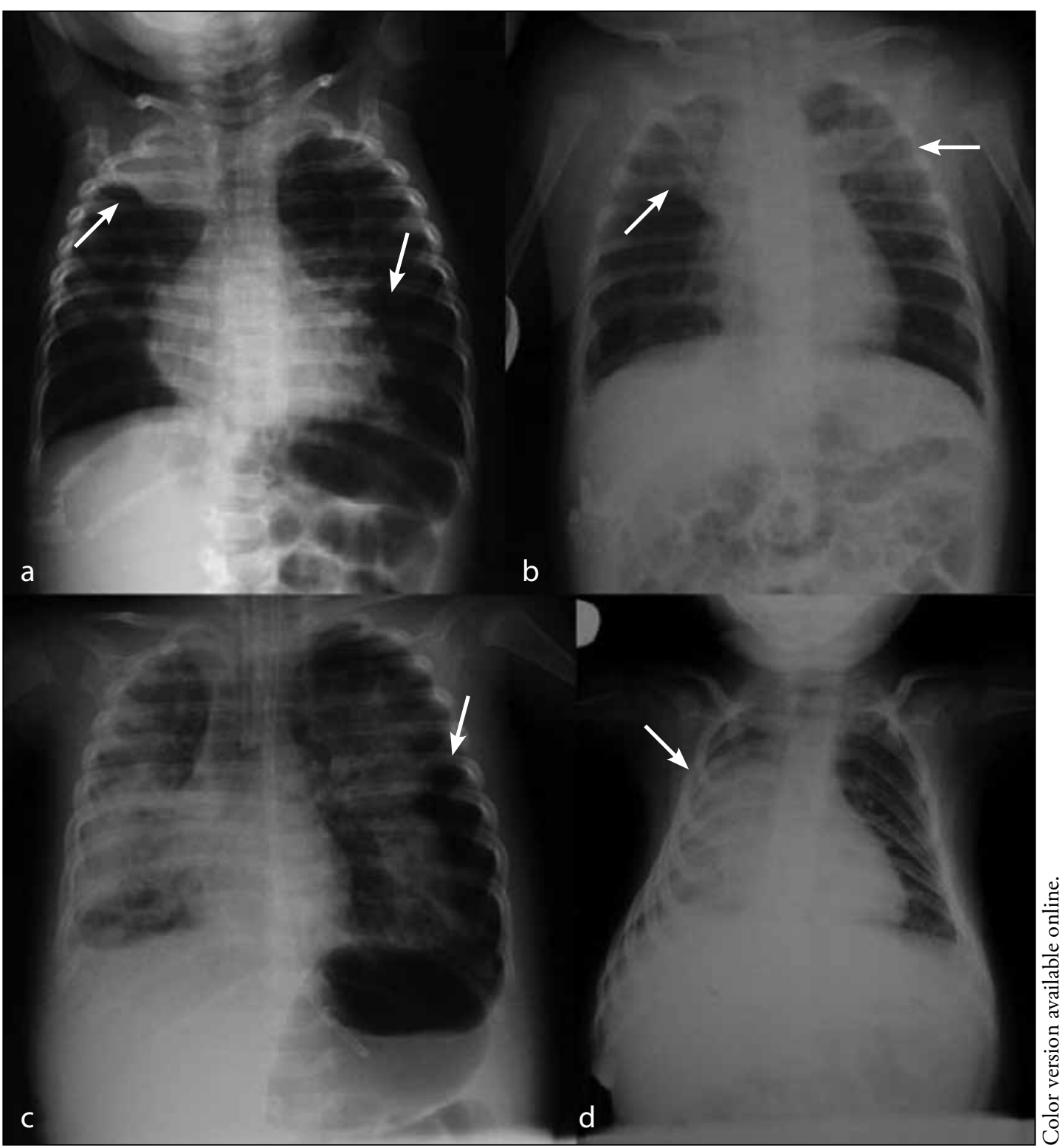

Fig. 3 Radiological presentations of bronchiolitis (a - atelectasis right superior lobe and patchy infiltrates on the left side; $b$-atelectasis right superior lobe and segmental atelectasis left and patchy infiltrates of the upper lobe; $c$ - respiratory distress and pneumothorax of the left side; $d$-atelectasis of the right side). 
leakage) were found in patients younger than 12 months with severe bronchiolitis during respiratory sintitial virus (RSV) seasons (Fig. 3). Patients with pathological CXRs and a severe form of the disease were RSV positive, which was proved by the rapid antigen test (RAT). There were a few patients with a negative RAT test, but with severe bronchiolitis, probably caused by other viruses.

\section{Chest radiograph in evaluation of wheezing in patients with other pulmonary and non-pulmonary diseases}

Sometimes diagnosis is unclear and therapy is unsuccessful, and a child can be under diag- nosed even in hospital. Many children with wheezing exhibit symptoms of bronchial obstruction caused by other, rare pulmonary or non-pulmonary diseases (Fig. 4 a, b, c, d).

Fig. 4a shows an CXR of a patient with intensive cough and wheezing. CXR revealed cystic formation on the right side, and serological analysis confirmed diagnosis of parasitic pulmonary infection caused by echinococcus.

Fig. $4 \mathrm{~b}$ presents a CXR of an infant who was admitted to hospital with a cough, increased respiratory rate, substernal and intercostals retractions, wheezing and diminished breath sounds over the right hemithorax and rhinorrhea. CXR showed a large noncalcified

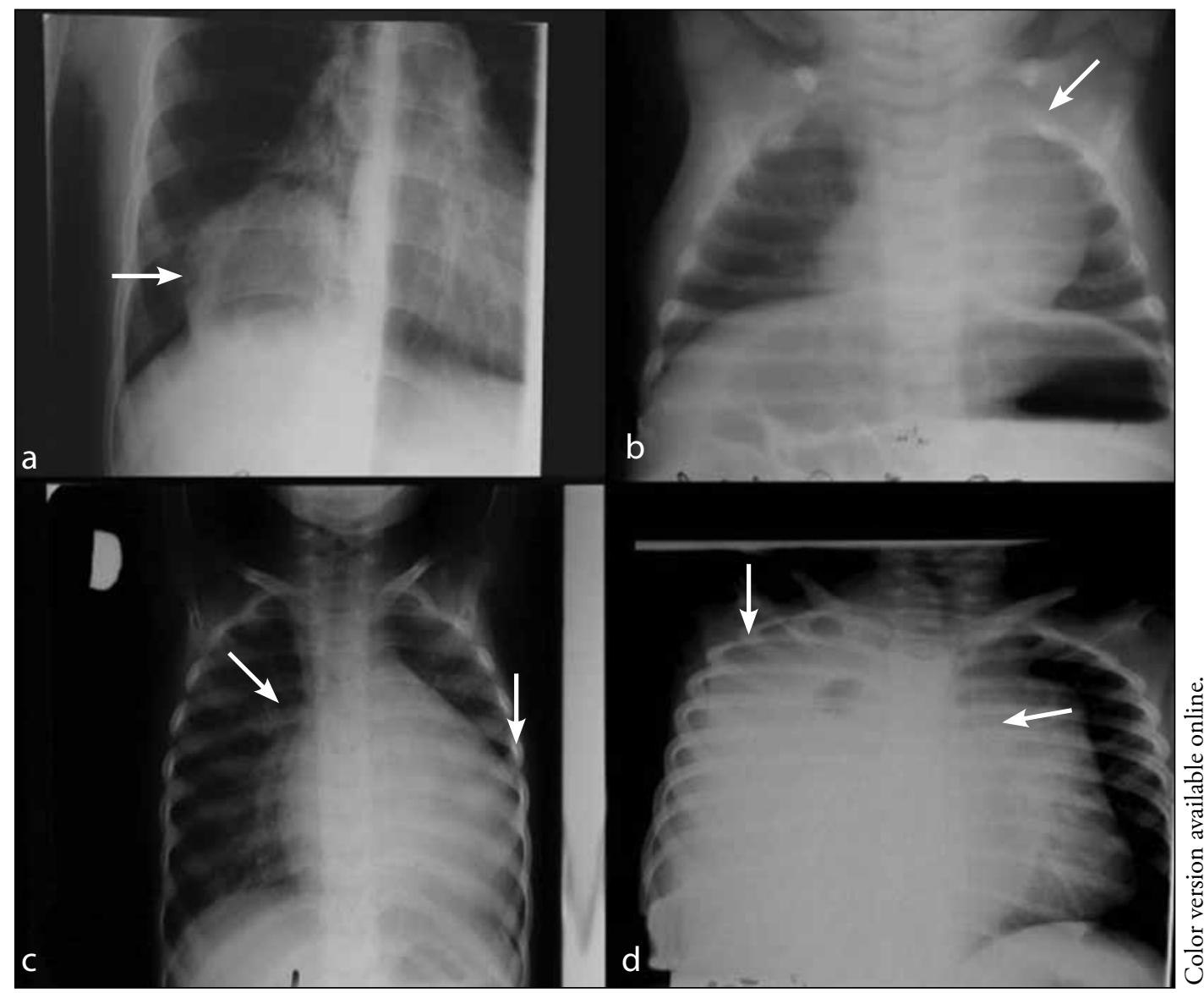

Fig. 4 Other pulmonary or non-pulmonary diseases (a - Right sided paravertebral cystic formation with thickened wall - cystic pulmonary formations: echinoccolal cystic formation; $b$ - wide mediastinum - hyperplasia of the thymus; $c$ enlarged heart with early signs of pulmonary edema - congestive heart disease (myocardiopathya); $d$-opacification of right thoracic side with enlarged mediastinum - malignant lymphoma). 
homogeneous mass in the mediastinum and left hemithorax. Additional imaging studies (ultrasound and magnetic resonance) proved hyperplasia of the thymus.

Fig. 4c is CXR of an 18 month old female with a history of recurrent wheezy episodes and with 3 previous hospitalizations in a regional hospital. She was unsuccessfully treated with parenteral corticosteroids and bronchodilatators. CXRs were not performed during those hospital stays. Upon admission to our hospital the initial CXR revealed an enlarged heart. Further diagnostics confirmed congestive heart disease and the child underwent a heart transplantation.

The patient presented in Fig. 4d was a young infant when the first episode of wheezing occurred. In the following 8 months he had several episodes of prolonged wheezing, without adequate response to therapy. This patient had no signs of infection, so radiological investigations were not performed. During his last hospital stay CXR revealed tracheal dislocation; complete shadowing of the right hemithorax with mediastinal and heart shift to the left side. Malignant lymphoma was diagnosed.

\section{Discussion}

Recurrent or severe wheeze is a common diagnostic and therapeutic problem, particularly in young children. Diagnostic issues may include the search for "mechanical" causes of wheeze, such as: structural airway narrowing, tracheobronchomalacia, compression by intrathoracic structures such as vessels, the heart, cysts, endobronchial tumors, or obstruction by a foreign body. Children with uncontrolled asthma or in acute asthma attacks, in some cases also need a radiological procedure (5).

Currently, no clinical parameter allows us to discern patients early in their disease. It is the purpose of this paper to discuss the place and utility of CXR in addressing the diagnostic issues outlined above.
Chest $\mathrm{X}$ ray is not required to diagnose asthma or to classify the severity of the disease. Severity assessments are clinical. Also CXR contributes little to defining the diagnoses of recurrent wheezing in preschool children. Children with uncomplicated asthma that respond appropriately to treatment do not require CXR (6). CXR is valuable in cases where there is a clinical suspicion of an alternative diagnosis, based on symptoms and signs of failure to respond to treatment. CXR can be useful in identifying complications of asthma (mucus plugging, atelectasis, air leakage) as we have presented in our patients.

Foreign body aspiration is a common cause of mortality and morbidity in childhood, especially among the population under 5 years. Once a diagnosis is suspected, CXR is invariably requested. This may provide clues to the diagnosis, but a normal CXR does not rule out FB aspiration. Plain CXR is useful for detection of a foreign body, since it may show obstructive emphysema, atelectasis, and pulmonary infiltration, or a radio opaque FB. Radiopaque FBs are easily localized, usually within a major airway.

However, it can be difficult to identify a non-radiopaque FB. In children younger than 3 years of age, in $80 \%$ of cases FBs are not visible on CXR (7). Radiological evaluation should start with AP and lateral views of the chest and neck. In some cases plain films may be interpreted as a normal, differential inflation of the affected lung, the most common abnormality identified, may be documented by CXR in the inspirium and expirium, CT of the thorax or bronchoscopy $(8,9,10,11)$. It has been reported that imaging studies have a sensitivity of $73 \%$ and a specificity of $45 \%$, however, up to $20 \%$ of patients will have both negative history and radiographic evaluation (12).

Misdiagnosis of FB aspiration may cause numerous and different symptoms. Persistent wheezing is a common symptom, often 
treated, in some cases for weeks or months, with bronchodilatators or corticosteroids. Sometimes stridor and respiratory distress are the main signs of FB aspiration. Coughing, wheezing and/or choking are present in $95 \%$ of cases $(13,14)$. Different clinical presentations can mimic the clinical picture of asthma, without therapeutic response or slow resolve pneumonia, bronchiectasis, lung abscess, pulmonary tuberculosis, etc. Findings on CXR can reveal secondary changes in the associated lung or pulmonary lobe, such as obstructive emphysema or overinflation of the lung or lobe distal to the airway obstruction. Additionally, the FB may shift in position. Thus, a previously suspicious radiographic study may be negative if it is repeated. One cannot assume that such a patient is now normal since a more likely explanation is that the FB has moved. Although appropriate radiological studies may localize the site of the foreign body, a significant number of children with retained airway FB have non-diagnostic films. In our series of patients with unrecognized, prolonged FB aspiration, all radiological findings were pathological, mostly with persistent infiltrative shadows or atelectatic regions. Obstructive emphysema, as an isolated finding, was characteristic in those patients where the FB was aspirated in a period shorter than 7-10 days.

The diagnosis of bronchiolitis is clinical. CXRs should not be used to diagnose bronchiolitis but occasionally may be warranted in infants and children where the diagnosis is uncertain, if respiratory distress or severe illness is present $(15,16)$. Radiology findings in individuals with bronchiolitis are variable and may include bronchial wall thickening, tiny nodules, linear opacities, atelectasis, patchy alveolar opacities, lobar consolidation and disturbance of ventilation (generalized or localized). While many infants or children with bronchiolitis have abnormalities on $\mathrm{CXR}$, there is conflicting evidence concern- ing whether this findings correlate well with disease severity. Studies suggest that in most cases of bronchiolitis, CXR offer no information that is likely to improve treatment and it may lead to inappropriate use of antibiotics. Among 153 children with acute bronchiolitis, Dawson et al. found no correlation between the degree of change on the chest radiograph and a clinical scoring method (17). However, in Shaw's 1991 study (18) of 213 infants with bronchiolitis, atelectasis was 2.7 times more likely to be found at presentation in the patients with severe disease than in those with mild disease. One survey showed that chest radiographs were obtained $83 \%$ of the time in patients with bronchiolitis, while antibiotics were prescribed only $69 \%$ of the time. Lobar or segmental consolidation is equally likely in patients with bronchiolitis with or without a bacterial pathogen (19). Current evidence does not support routine radiography in children with bronchiolitis $(20,21)$.

Mediastinal masses (i.e., tumors, bronchogenic cysts, enlarged lymph nodes, hyperplasic thymus, different kinds of diaphragmatic hernia) can compress the trachea or bronchi, thereby leading to stridor or wheezing. The CXR is usually abnormal, and patients are generally unresponsive to bronchodilators. The thymus is normally situated in the anterior mediastinum and involutes during the first 3 to 4 years of life. It has been reported that the thymus gland increases in size as a physiological response to stress, and that this is common cause of thymic rebound in infants. Appropriate investigation involves adequate radiological imaging, where CXR has a significant role as an initial diagnostic procedure.

Children with severe heart disease in time develop heart failure. Congestive heart failure $(\mathrm{CHF})$ means the heart is unable to provide an adequate blood supply to the rest of the body. When and how it develops depend on the type of underlying heart disease. In the 
early stages of congestive heart failure, a person may have no symptoms. Wheezing may be an isolated physical sign for a period of a few months or longer, but other diagnoses must be suspected in all patients with persistent wheezing, or with prolonged complains. Patients have shortness of breath, difficulty breathing when laying flat, and swelling in the lower extremities. Heart murmurs or cardiomegaly, as well as cyanosis without respiratory distress, may suggest cardiac disease. Chest radiography, echocardiography, angiography or a combination of these diagnostic tests may be appropriate. Unlike wheezing in asthma patients, CHF patients will often have a large heart on chest $x$-ray (2). Our patient with CHF had isolated wheezing, without other signs of heart disease, and CXR was not performed during the outpatients visits or two previous hospital treatments. Most children with solitary pulmonary nodules or cysts do not experience symptoms. Generally, these are detected as an incidental finding. A pulmonary nodule must grow to at least $1 \mathrm{~cm}$ in diameter before it can be seen on a CXR film. Chest x-ray films can provide information regarding size, shape, cavitations, growth rate, and calcification pattern. All of these features can help determine whether the lesion is benign or malignant. However, none of these features is entirely specific for lung malignancy. Pulmonary hydatid disease affects the right lung in almost $60 \%$ of cases (22). Most intact lung cysts are discovered incidentally on the CXR, but occasionally unruptured cyst results in a cough, wheezing, haemoptysis and/or chest pain. Symptomatic hydatic disease of the lung, however, most often follows the rupture of the cyst, which can cause the sudden onset of an intensive cough, fever and deterioration of the child's condition. In these cases, CXR is necessary. One of the patients presented here had a soli- tary lesion in the right lower lobe; another child had two cysts in both sides of the lungs. In both cases the health of the children was generally good and the CXR result was unexpected.

CXRs are frequently obtained in the evaluation of wheezing disorders in children. An efficient and cost-effective approach would be to forego CXRs when a clinical diagnosis is certain, and to perform them only if a complication is suspected or if the etiology of the respiratory illness is uncertain. Despite the fact that the place of CXR in the diagnostic algorithm in children with asthma and bronchiolitis is not so important, there are other numerous conditions, with persistent/ recurrent wheezing as a dominant symptom. In these cases CXRs findings are valuable and can reveal different pathological features valuable for proper diagnosis.

The decision to perform a CXR in a child with wheezing should be closely planned and based on careful clinical evaluation. The value of CXRs is in revealing complications or alternative causes of wheezing but it is of minor importance in evaluation of wheezing episodes in patients with asthma. Typically it is not recommended to repeat a CXR in a patient with documented asthma, unless a new pathological process is suspected. Although the increased risk of cancer is extremely low with a single CXR, the risks are cumulative and therefore even a CXR should be ordered judiciously to minimize any long-term risks.

\section{Conclusion}

In conclusion, an efficient approach in patients with wheezing would be to forego CXR when the clinical diagnosis is certain, and to perform them only if a complication is suspected or if the etiology of the respiratory illness is uncertain. 
Conflict of interest: The authors declare that they have no conflict of interest.

\section{References}

1. Chin SE. Pediatric Reactive Airway Disease. [Internet]. 2010 [Updated 2010 Apr 6; cited 2011 May 12]. Available at : http://emedicine.medscape. com/article/800119-overview.

2. Mody AP, Mahmoud G. Difficulty Breathing In Infants And Young Children: An Evidence-Based Approach. Pediatric Emergency Medicine Practice [serial on the Internet]. 2004 Oct [cited 2011 May 12];1(3):[20 p.]. Available from: www.ebmedicine. net/topics.php? paction=show TopicSeg.

3. World Health Organization. Pneumonia Vaccine Trial Investigators' Group. Standardization of interpretation of chest radiographs for the diagnosis of pneumonia in children. Geneva: World Health Organization; 2001

4. Roback M, Dreitlein DA. Chest radiograph in the evaluation of first time wheezing episodes: Review of current clinical practice and efficacy. Pediatric Emergency Care. 1998;14:181-4.

5. Bacharier LB, Boner A, Carlsen KH, Eigenmann PA, Frischer T, Götz M, et al. Diagnosis and treatment of asthma in childhood: a Practicall consensus report. Allergy 2008;63(1):5-34.

6. National Asthma Council Australia. Asthma Management Handbook 2006. Melbourne (Australia): National Asthma Council Australia LTD; 2006.

7. Deskin R, Young G, Hoffman R. Management of pediatric airway foreign bodies. Laryngoscope. 1997;107(4):540-43.

8. Hong WS, Im SA, Kim HL, Yoon J. CT evaluation of airway foreign bodies in children: emphasis on the delayed diagnosis and differentiation from airway mucus plugs. Japanese Journal of Radiology. 2013;31(1):31-8.

9. Bai W, Zhou X, Gao X, Shao C, Califano JA, Ha PK. Value of chest CT in the diagnosis and management of tracheobronchial foreign bodies. Pediatrics International. 2011;53:515-8.

10. Korlacki W, Korecka K, Dzielicki J. Foreign body aspiration in children: diagnostic and thera- peutic role of bronchoscopy. Pediatr Surg Int. 2011;27:833-7.

11. Midulla F, Guidi R, Barbato A, Forenza N, Marseglia $\mathrm{G}$, Pifferi $\mathrm{M}$, et al. Foreign body aspiration in children. Pediatrics International. 2005;47:663-8.

12. Freidman EM. Update on the Pediatric Airway: Tracheobronchial Foreign Bodies. Otolaryngol Clin North Am. 2000;33(1):179-85.

13. Reilly J, Thompson J, MacArthur C, Pransky $\mathrm{S}$, Beste D, Smith M, et al. Pediatric aerodigestive foreign body injuries are complications related to timeliness of diagnosis. Laryngoscope. 1997;107:17-20.

14. Darrow DH, Holinger LD. Aerodigestive tract foreign bodies in the older child and adolescent. Ann Otol Rhinol Laryngol. 1996;105:267-71.

15. American Academy of Pediatrics, Steering Committee on Quality Improvement and Management. Classifying recommendations for clinical practice guidelines. Pediatrics. 2004;114 :874-7.

16. Bordley WC, Viswanathan M, King VJ, Sutton SF, Jackman AM, Sterling L, et al. Diagnosis and testing in bronchiolitis: a systematic review. Arch Pediatr Adolesc Med. 2004;158:119-26.

17. Dawson KP, Long A, Kennedy J, Mogridge N. The chest radiograph in acute bronchiolitis. J Paediatr Child Health. 1990;26(4):209-11.

18. Shaw KN, Bell LM, Sherman NH. Outpatient assessment of infants with bronchiolitis. Am J Dis Child. 1991;145(2):151-5.

19. Friis B, Eiken M, Hornsleth A, Jensen A. Chest $\mathrm{X}$-ray appearances in pneumonia and bronchiolitis. Correlation to virological diagnosis and secretory bacterial findings. Acta Paediatr Scand. 1990;79(2):219-25.

20. Martinez FD. Respiratory syncytial virus bronchiolitis and the pathogenesis of childhood asthma. Pediatr Infect Dis J. 2003;22(2 suppl):S76-S82.

21. Mahabee-Gittens EM, Grupp-Phelan J, Brody AS, Donnelly LF, Bracey SE, Duma EM, et al. Identifying children with pneumonia in the emergency department. Clin Pediatr (Phila). 2005;44(5):427-35.

22. Aribas OK, Kanat F, Gomus N, Turk E. Pleural complications of hydatic disease. J Thorac Cardiovasc Surg. 2002;123:492-7. 